\title{
Processos perceptuais e cognitivos na leitura de palavras: propriedades dos movimentos oculares
}

\author{
Processos perceptuais e cognitivos na leitura de palavras
}

\author{
Elizeu Coutinho de Macedo \\ Katerina Lukasova \\ Juliana Emy Yokomizo \\ Lívia Carolina Ariente \\ Juliana Koakutu \\ José Salomão Schwartzman
}

\section{Resumo}

A análise do padrão do movimento ocular auxilia discriminar leitores competentes daqueles com dificuldades de leitura. $\bigcirc$ padrão de leitura dos movimentos oculares é afetado por propriedades psicolingüísticas da palavra, tais como: comprimento, regularidade, freqüência e lexicalidade $O$ objetivo do presente estudo foi analisar o padrão de movimentos oculares durante a leitura em português do Brasil e compará-lo com resultados de estudos de outras línguas. Os movimentos oculares durante a leitura de palavras e pseudopalavras foram analisados em 20 universitários com idade média de 20,5 anos $(D P=2,9)$. Os resultados indicam que o número de fixações, tempo da primeira fixação e tempo total das fixações foram influenciados pelas variáveis, comprimento, lexicalidade e freqüência dos itens. Foi observado efeito de regularidade para todas as variáveis, exceto naquela do número de fixações. Os resultados obtidos foram semelhantes àqueles encontrados em outras línguas com predomínio de palavras regulares, mas não de palavras irregulares, como o inglês.

Palavras-chave: leitura; movimentos oculares; linguagem; avaliação.

\section{Perceptual and cognitive processes in word reading: eye movement characteristics}

\begin{abstract}
Analyses of eye movement's pattern contribute significantly to the discrimination between good and poor readers. The pattern of eye movements during reading is affected by psycholinguistic properties of words such as: length, regularity, and frequency. The objective of the present study was to analyze eye movements during words and non-words reading in Brazilian Portuguese and compare the results with the data from other languages. Eye movements during the words and non-words reading were recorded from 20 university students with the mean age of 20.5 years $(S D=2.9)$. Results showed significant effect of word length, regularity and frequency on number of fixations, first fixation duration and gaze duration. Regularity showed effect on all of the variables except on number of fixations. The results are similar to those found in languages with predominantly regular orthographies, but not with the irregular ones such as English.
\end{abstract}

Keywords: reading; eye movements; language; measurement.

\section{Procesos perceptivos y cognitivos en la lectura de palabras: propiedades de los movimientos oculares}

\begin{abstract}
Resumen
El análisis del padrón del movimiento ocular ayuda a discriminar lectores competentes de aquellos con dificultades de lectura. El padrón de lectura de los movimientos oculares es afectado por propiedades psico-lingüísticas de la palabra, como por ejemplo: extensión, regularidad, frecuencia y lexicalidad. El objetivo de este estudio fue analizar el padrón de movimientos oculares durante la lectura en portugués del Brasil y compararlo con resultados de estudios de otros idiomas. Durante la lectura, los movimientos oculares de palabras y pseudo-palabras fueron analizados en 20 universitarios con un promedio de edad de 20,5 años ( $D P=2,9)$. Los resultados indican que el número de fijaciones, tiempo de la primera fijación y tiempo total de las fijaciones fueron influenciados por las variables extensión, lexicalidad y frecuencia de los ítems. Fue observado efecto de regularidad para todas las variables, excepto en aquella del número de fijaciones. Los resultados obtenidos fueron semejantes a los encontrados en otros idiomas con predominancia de palabras regulares pero no de palabras irregulares, como el inglés.

Palabras clave: lectura; movimientos oculares; lenguaje; evaluación.
\end{abstract}




\section{Introdução}

Avaliações padronizadas de desempenhos comportamentais durante a leitura produziram uma extensa literatura sobre o reconhecimento de palavras. Tipicamente, nestes estudos, têm sido usadas medidas de tempo de reação em tarefas de decisão lexical (Balota \& Chumbley, 1984), provas de categorização semântica (Balota, 1990), nomeação de palavras (Macedo, 1999; Pulvermüller, Assadollahi \& Thomas, 200I) e leitura auto-monitorada (Kello \& Plaut, 2003). O registro de movimento ocular tem sido usado em diferentes paradigmas como nas provas de leitura de palavras isoladas (Blanchard, Pollatsek \& Rayner, 1989), nos textos dinâmicos em que as palavras são precedidas ou seguidas de uma máscara (Sereno \& Rayner, 1992) e na apresentação de sentença (McConkie \& Rayner, 1975).

As principais propriedades do movimento ocular, analisadas nos estudos de leitura são as fixações e os movimentos sacádicos. As fixações são breves períodos de tempo durante os quais o olho permanece examinando uma pequena área do estímulo. $O$ movimento que o olho executa para a área de fixação é chamado de sacada. A função principal da fixação é analisar detalhadamente o texto no campo foveal, onde a informação é mais facilmente passível de ser obtida, ao contrário das regiões parafoveal e periférica (Rayner, 1998).

Durante a leitura, os movimentos sacádicos e fixações adquirem um determinado padrão que difere do observado em outros tipos de tarefas como no processamento de paisagens ou objetos (Boyce \& Pollatsek, 1992), mas semelhante ao de leitura de partitura musical (Land, 2004). Embora a leitura pareça ser um processo fluido e contínuo, na realidade não o é. As fixações acontecem somente sobre algumas palavras do texto, sendo que as palavras curtas com 2 a 3 letras são geralmente omitidas, enquanto que as maiores podem ser fixadas mais de uma vez (Rayner, 1998). Embora nem todas as palavras sejam fixadas, todas recebem algum tipo de processamento visual, pois se as palavras não-fixadas durante a leitura de uma frase forem excluídas e a frase for apresentada novamente, $\mathrm{o}$ texto se tor- nará incompreensível para este leitor (Rayner e cols., 1996).

$\mathrm{Na}$ leitura de textos em voz alta, a fixação dura cerca de 275 milésimos de segundos e o comprimento médio de sacadas é de 6 letras. No entanto, antes da realização de uma sacada propriamente dita, pode-se identificar um breve período de planejamento, chamado de latência sacádica e dura aproximadamente de 150 a 175 milésimos de segundos (Abrams \& Jonides, 1988; Rayner, Slowiazete, Clifton \& Bertera, 1983; Salthouse \& Ellis, 1980; Salthouse, Ellis, Diener \& Sonberg, 198I). A variação na latência afeta a acurácia da localização da sacada ao alvo a ser lido, o que sugere que a programação da sacada é feita em paralelo com o processo de compreensão de leitura (Jacobs, 1987; Nazir \& Jacobs, 1991).

Nas línguas ocidentais, o movimento ocular durante a leitura normalmente ocorre da esquerda para direita. No entanto, alguns movimentos sacádicos podem ser observados no sentido inverso, ou seja, da direita para a esquerda. Esses tipos de movimentos se chamam sacadas regressivas e acontecem em 10 a $15 \%$ de vezes durante a leitura (Starr \& Rayner, 200I). Sua função é re-fixar a palavra a fim de uma nova inspeção, sendo que as sacadas curtas numa mesma palavra denotam um problema no posicionamento da fixação, enquanto as longas mostram dificuldade no processamento da palavra. As sacadas regressivas, maiores que dez letras, refletem a dificuldade que o leitor tem na compreensão do conteúdo. Murray e Kennedy (1998) observaram diferença entre bons e maus leitores com relação à precisão das sacadas regressivas. Os bons leitores localizaram a área alvo com poucas sacadas de alta precisão, já os maus leitores apresentaram pequenas sacadas regressivas em busca da área alvo.

Millet e Sparrow (2004) mostraram que o processo de leitura em bons leitores se inicia antes mesmo da primeira fixação e não se dá de forma seqüencial. Hyönä e Olson (1995) mostraram que a fixação inicial geralmente acontece na primeira metade da palavra. Caso a palavra seja processada com sucesso, o olho é movido para outra palavra; no caso de insucesso, uma segunda fixação de maior duração 
é efetuada perto do final da palavra. As informações que são extraídas antes da primeira fixação estão relacionadas com propriedades físicas e lingüísticas tais como comprimento da palavra e freqüência de ocorrência da palavra na língua.

O comprimento da palavra determina a localização da primeira fixação e também o número de fixações dentro da palavra (Pollatsek \& Rayner, 1982). Bons leitores tendem a ajustar, aumentar ou diminuir a amplitude da sacada de acordo com a amplitude da palavra aumentando a velocidade de leitura (O'Regan, 1980). Em maus leitores, essa capacidade foi encontrada limitada em palavras com mais de cinco letras (MacKeben e cols., 2004). Já a freqüência da palavra é determinada através da contagem de sua ocorrência na forma impressa de uma determinada língua. Assim, palavras de alta freqüência são aquelas com alto índice de ocorrência, sendo mais familiares aos leitores. A maior familiaridade com a palavra assim como a maior previsibilidade contextual, leva à diminuição na duração e no número de fixações (Monaghan \& Ellis, 2002; Rayner, 1998).

Estudos nos últimos 70 anos mostram que há uma correlação entre o aumento da habilidade de leitura e eficácia no padrão de movimento ocular. O tamanho das sacadas aumenta de acordo com a melhora nas habilidades de leitura, enquanto a duração da fixação, o número de fixações e a freqüência de sacadas regressivas diminuem (Buswell, 1922). McConkie e colaboradores (I99I) realizaram estudos na língua inglesa e demonstraram que o comportamento do movimento ocular em crianças apresenta maior variabilidade do que em adultos; no entanto, ambos tendem a normatizar a localização onde o olhar é fixado, dirigindo-o para a parte central da palavra. Essa normatização do padrão de movimentos oculares ocorre com o progresso na aquisição de habilidades de leitura e escrita.

O objetivo do presente trabalho foi verificar se o padrão de movimentos oculares durante a leitura de palavras e pseudopalavras de bons leitores da língua portuguesa apresenta as mesmas características já encontradas em outros idiomas.

\section{Método}

\section{Participantes}

Foram avaliados 20 universitários, sendo metade da cada sexo e com idade média de 20 anos e 6 meses $(D P=2,9)$. Nenhum dos participantes relatou, na entrevista inicial, ter tido dificuldades para aprender a ler e escrever. Também não foram observados problemas de leitura de familiares diretos: pais e irmãos. Os participantes relataram ler em média 6,2 livros por ano. Todos os sujeitos apresentavam visão normal ou corrigida com pontuação acima de 0,8 na escala Snellen.

\section{Instrumento e equipamento}

Foram aplicadas as provas de Leitura de Palavras Isoladas e Leitura de Pseudopalavras. A Prova de Leitura de Palavras é composta por 96 itens, sendo que 72 são palavras-alvo e 24 são palavras para controle do início e fim do registro de movimento ocular. As palavras foram apresentadas em 12 listas de 8 palavras posicionadas em duas colunas de 4 palavras cada. As palavras foram selecionadas em função das seguintes variáveis psicolíngüísticas: freqüência, regularidade e tamanho. A freqüência foi obtida a partir da lista de freqüência de palavras da Associação Brasileira de Dislexia (ABD) e aferidas com as do Corpus NILC da Universidade de São Carlos (www.linguateca.pt). Com relação à regularidade, as palavras foram classificadas como: regulares, regras e irregulares. Nas palavras regulares, cada letra corresponde a apenas um som e vice-versa (por exemplo, a palavra pata); nas palavras do tipo regra, a correspondência letra-som é regulada por regras de posição (por exemplo, a palavra casa, em que a letra soa como /z/ porque está entre duas vogais); e nas irregulares a correspondência não segue regra alguma, sendo a leitura totalmente arbitrária (por exemplo, a palavra exército, em que a letra $x$ soa como /z/) (Lemle, 1991). Por fim, o comprimento foi analisado da seguinte forma: palavras curtas de 3 a 5 letras; médias de 6 a 8 letras e longas de 10 a 14 letras. Assim, a lista final das palavras alvo (72) contém: 24 palavras regulares, 24 irregulares e 24 regra, sendo 12 de cada uma delas de alta e 12 de 
baixa freqüência. Destas, 4 são curtas, 4 médias e 4 longas.

A Prova de Leitura de Pseudopalavras é composta por 48 palavras, sendo que 36 são palavras-alvo e 12 para controle. A disposição dos itens foi a mesma da Lista de Palavras. As pseudopalavras foram criadas a partir das palavras de alta freqüência na lista de prova de palavras isoladas com as seguintes modificações: para as palavras curtas foi modificada uma letra, para as médias duas e, para as longas três letras. Foram modificadas preferencialmente as consoantes, sendo a substituição feita dentro de mesmo grupo sonoro (Russo \& Behlau, 1993), sem alterar as demais variáveis psicolingüísticas.

As listas foram apresentadas na tela de um computador, com o equipamento EyeGaze ${ }^{\circledR}$ desenvolvido pela LC Technologies Inc. e registra os movimentos oculares. O programa Trace-CronoFonos 1.0 (Macedo, Lukasova, Capovilla, Capovilla \& Schwartzman, 2005) foi usado para análise dos parâmetros de movimentos oculares e para criação de tabelas com os seguintes dados: localização e duração de fixações, tempo de início da cada fixação e duração das sacadas. O programa também registra, em tempo real, as locuções produzidas pelos leitores durante a avaliação.

\section{Procedimento}

Inicialmente foram conduzidas entrevistas para verificar hábitos de leitura e histórico de possíveis dificuldades de aprendizagem. Os sujeitos que leram pelo menos 5 livros no último ano e sem histórico familiar de problemas de leitura, assinaram o termo de consentimento livre e esclarecido aprovado pelo comitê da Universidade Presbiteriana Mackenzie. Em seguida foram submetidos aos testes de leitura no computador. As avaliações tiveram duração média de vinte minutos. As aplicações foram individuais e cada sujeito era posicionado a uma distância de 50 centímetros da tela do computador. Foi usada uma mesa oftalmológica (LF-M4 da DFV S/A) com apoio da cabeça e uma queixeira a fim de evitar movimentos amplos de cabeça. $O$ equipamento foi calibrado para cada sujeito. A apresentação dos estímulos foi feita de forma automática, com o tempo de exposição dependente do desempenho do próprio sujeito. A fim de controlar a posição inicial do olho e indicar o aparecimento de uma nova tela, um ponto intermitente era apresentado, entre as listas de palavras, no canto superior esquerdo da tela por 800 milésimos de segundos.

\section{Resultados}

Os movimentos oculares durante a leitura dos itens foram analisados em função das seguintes variáveis psicolingüísticas: lexicalidade-frequência (alta freqüência, baixa freqüência e pseudopalavra), comprimento (curta, média e longa) e regularidade (regular, regra e irregular). As variáveis dependentes analisadas foram: Número de Fixações (NF), Tempo da Primeira Fixação na palavra (TPF) e Tempo de Fixação Total na palavra (TFT).

ANOVA de medidas repetidas revelou efeito principal para lexicalidade-freqüência no NF $(F[2,38]=83,658 ; p<0,000)$. Análises Post Hoc (Bonferroni) revelaram diferença entre os tipos de palavras, sendo que o NF nas palavras de alta freqüência foi menor do que palavras de baixa freqüência, e estas menores do que as pseudopalavras. Diferença significativa foi encontrada também para TPF $(F[2,38]=138,25 ; p<0,000)$, com média crescente na seguinte ordem: palavras de alta freqüência; baixa frequiência e pseudopalavras. $O$ mesmo padrão foi encontrado para TFT $(F[2,38]=207,64 ; p<0,000) \mathrm{com}$ tempo total de fixação crescente pelas categorias: alta freqüência; baixa freqüência e pseudopalavras. A Tabela I sumariza os valores médios e desvio-padrão dos valores obtidos em função da lexicalidadefreqüência.

O efeito de comprimento também foi analisado através de ANOVA de medidas repetidas. Resultados mostram que o NF variou nos três grupos, tanto para as palavras $(F[2,38]=247,32 ; p<0,000)$ quanto para as pseudopalavras $(F[2,38]=222,05$; $p<0,000)$. As médias de NF seguem uma curva crescente para os dois testes na seguinte ordem: curta, média e longa. O TPF variou tanto para o comprimento como para as palavras $(F[2,38]=24,39$; 
Tabela I. Valores de média e desvio-padrão para Número de Fixação (NF), Tempo da Primeira Fixação (TPF) e Tempo de Fixação Total (TFT) em milésimos de segundos em função da lexicalidadefreqüência.

\begin{tabular}{|c|c|c|c|c|c|c|}
\hline & Alta Freqüência & Baixa Freqüência & Pseudopalavra & $F(2,38)$ & $p$ & Post Hoc \\
\hline \multirow{2}{*}{ NF } & 1,94 & 2,26 & 2,85 & \multirow{2}{*}{83,66} & \multirow{2}{*}{0,000} & \multirow{2}{*}{$a, b, c$} \\
\hline & $(0,27)$ & $(0,30)$ & $(0,49)$ & & & \\
\hline TPF (ms) & $\begin{array}{r}464,7 \\
(88,49)\end{array}$ & $\begin{array}{l}506,11 \\
(100,1)\end{array}$ & $\begin{array}{r}734,12 \\
(103,33)\end{array}$ & 138,25 & 0,000 & $a, b, c$ \\
\hline TFT (ms) & $\begin{array}{r}790,91 \\
(166,98)\end{array}$ & $\begin{array}{r}967,32 \\
(175,33)\end{array}$ & $\begin{array}{r}1715,00 \\
(325,90)\end{array}$ & 207,64 & 0,000 & $a, b, c$ \\
\hline
\end{tabular}

Comparações Bonferroni: a) Palavras de Alta freqüência versus Palavras de Baixa; b) Palavras de Alta freqüência versus Pseudopalavras; c) Palavras de Baixa freqüência versus Pseudopalavras.

$p<0,000)$ quanto para as pseudopalavras $(F[2,38]=10,41 ; p<0,000)$. No entanto, análises Pos Hoc indicaram não haver diferença entre os itens de comprimento curto e médio para nenhum dos dois tipos de itens. De maneira similar, foi observada diferença significativa no TFT em função do comprimento tanto para as palavras $(F[2,38]=169,4$; $p<0,000)$ quanto para as pseudopalavras $(F[2,38]=173,75 ; p<0,000)$. Como no caso de NF, as médias de TFT seguem uma curva crescente. A
Tabela 2 apresenta os valores dos três parâmetros dos movimentos oculares em função do comprimento para as palavras e pseudopalavras.

A Figura I ilustra os traçados dos movimentos oculares de um mesmo sujeito lendo palavras e pseudopalavras. Os traços retos ilustram os movimentos sacádicos, e os círculos as fixações. A duração das fixações é proporcional ao diâmetro dos círculos.

$O$ efeito de regularidade também foi analisado por meio de ANOVA de medidas repetidas. O NF não foi

Tabela 2. Valores de média e desvio-padrão para Número de Fixação (NF), Tempo da Primeira Fixação (TPF) e Tempo de Fixação Total (TFT) em milésimos de segundos em função do comprimento e do tipo do item: palavras e pseudopalavras

\begin{tabular}{|c|c|c|c|c|c|c|c|}
\hline & & curta & média & longa & $F(2,38)$ & $p$ & Post hoc \\
\hline \multirow{3}{*}{ Palavras } & $\mathrm{NF}$ & $\begin{array}{r}1,42 \\
(0,18)\end{array}$ & $\begin{array}{r}1,69 \\
(0,19)\end{array}$ & $\begin{array}{r}3,18 \\
(0,52)\end{array}$ & 247,32 & 0,000 & $a, b, c$ \\
\hline & $\begin{array}{l}\text { TPF } \\
(\mathrm{ms})\end{array}$ & $\begin{array}{r}541,91 \\
(117,63)\end{array}$ & $\begin{array}{r}519,54 \\
(111,06)\end{array}$ & $\begin{array}{r}392,35 \\
(101,13)\end{array}$ & 24,39 & 0,000 & $\mathrm{~b}, \mathrm{c}$ \\
\hline & $\begin{array}{l}\text { TFT } \\
(\mathrm{ms})\end{array}$ & $\begin{array}{r}724,41 \\
(155,03)\end{array}$ & $\begin{array}{r}789,4 \\
(166,2)\end{array}$ & $\begin{array}{r}1123,05 \\
(202,24)\end{array}$ & 169,4 & 0,000 & $a, b, c$ \\
\hline \multirow{3}{*}{ Pseudopalavras } & NF & $\begin{array}{r}1,61 \\
(0,34)\end{array}$ & $\begin{array}{r}2,52 \\
(0,56)\end{array}$ & $\begin{array}{r}4,42 \\
(0,81)\end{array}$ & 222,05 & 0,000 & $a, b, c$ \\
\hline & $\begin{array}{l}\text { TPF } \\
(\mathrm{ms})\end{array}$ & $\begin{array}{r}731,87 \\
(175,27)\end{array}$ & $\begin{array}{r}833,75 \\
(153,81)\end{array}$ & $\begin{array}{r}637,88 \\
(120,77)\end{array}$ & 10,41 & 0,000 & $\mathrm{~b}, \mathrm{c}$ \\
\hline & $\begin{array}{l}\text { TFT } \\
\text { (ms) }\end{array}$ & $\begin{array}{l}1112,22 \\
(276,42)\end{array}$ & $\begin{array}{c}1635,66 \\
(359,92)\end{array}$ & $\begin{array}{r}2393,56 \\
(454,13)\end{array}$ & 173,75 & 0,000 & $a, b, c$ \\
\hline
\end{tabular}

Comparações Bonferroni: a) itens curtos versus médio; b) itens curtos versus longo; c) itens médios versus longo. 


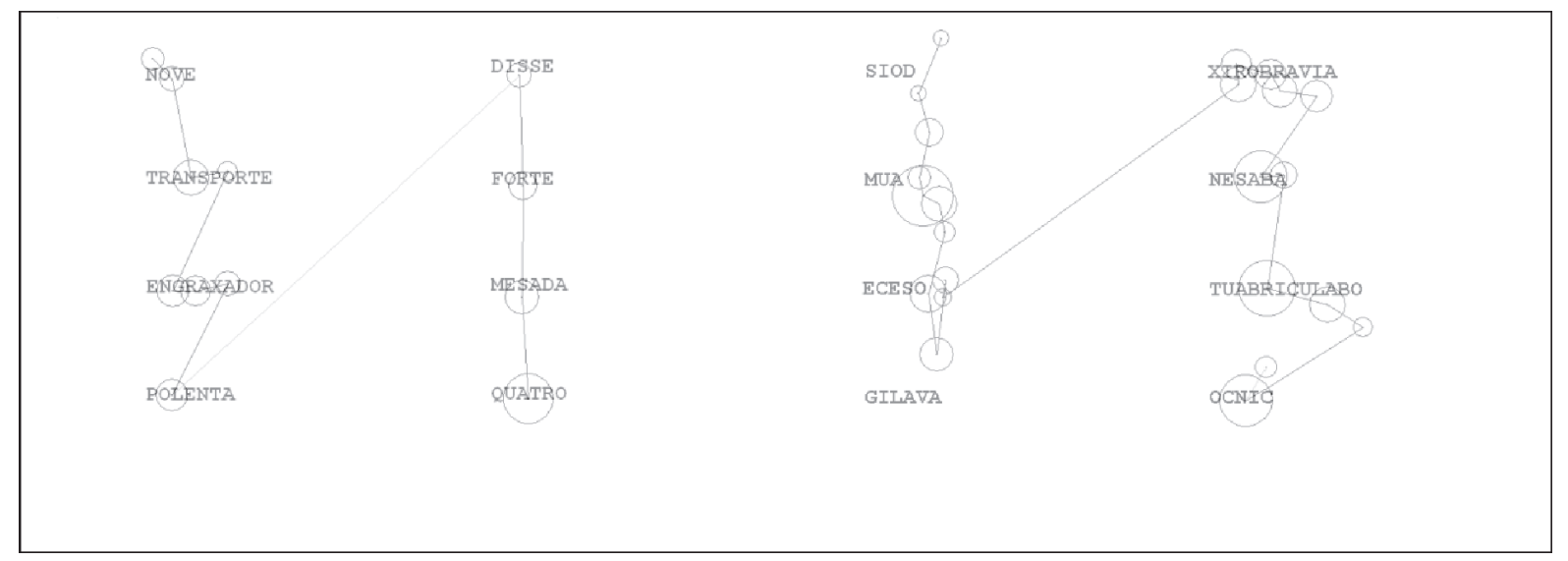

Figura I. Traçados dos movimentos oculares durante a leitura da lista de palavras (esquerda) e de pseudopalavras (direita)

afetado pela regularidade dos itens. Foram observadas diferenças significativas tanto para $\circ$ TPF $(F[2,38]=4,148 ; p=0,023)$ quanto para $\circ \mathrm{TFT}$ $(F[2,38]=10,642 ; p=0,000)$. Tais diferenças podem ser atribuídas principalmente aos itens irregulares que demandaram mais tempo para as fixações. A Tabela 3 apresenta os valores de média e desvio-padrão para os parâmetros de movimentos oculares em função da regularidade dos itens.

\section{Discussão}

Os resultados gerais obtidos nesse estudo inédito no Brasil e em língua portuguesa podem ser comparados com aqueles já encontrados em outras línguas e países. Assim, o tempo da primeira fixação e tempo de fixação total aumentou de acordo com a familiaridade do leitor com a palavra, sendo este mais curto nas palavras de alta freqüência e mais longo nas pseudopalavras. Aumento do tempo indica que o movimento ocular durante a leitura em português, como seria de esperar, também sofre efeito de freqüência encontrado em outras línguas (Rayner, 1998).

O tempo da primeira fixação reflete o acesso lexical, já o tempo de fixação total representa processo de integração contextual (Inhoff, 1984). De acordo com Sereno e Rayner (2003), a informação visual começa a ser processada 60 milésimos de segundos após o início da primeira fixação e o acesso lexical ocorre dentro dos primeiros 100 a 200 milésimo de segundos da fixação.

$\mathrm{Na}$ ausência de um contexto, como foi o caso do presente estudo, pode se presumir que o tempo de fixação total reflete o processamento lexical e se-

Tabela 3. Valores de média e desvio padrão para Número de Fixação (NF), Tempo da Primeira Fixação (TPF) e Tempo de Fixação Total (TFT) em milésimos de segundos em função da regularidade

\begin{tabular}{crrrccc}
\hline & Regular & Regra & Irregular & $F(2,38)$ & $p$ & p.h. \\
\hline \multirow{2}{*}{ NF } & 2,132 & 2,065 & 2,081 & 0,572 & 0,569 & \\
& $(0,296)$ & $(0,278)$ & $(0,358)$ & & & \\
\hline \multirow{2}{*}{ TPF (ms) } & 474,733 & 465,599 & 513,787 & & & \\
& $(92,26)$ & $(98,126)$ & $(117,90)$ & 4,148 & 0,023 & b \\
\multirow{2}{*}{ TFT (ms) } & 869,700 & 841,960 & 926,294 & 10,642 & 0,000 & b.c. \\
& $(168,96)$ & $(162,163)$ & $(184,542)$ & & & \\
\hline
\end{tabular}

Comparações Bonferroni: a) regulares versus regra, b) regulares versus irregular, c) regra versus irregular. 
mântico integrando também o tempo da vocalização de palavra. Com base nestes dados, algumas hipóteses podem ser levantadas acerca dos resultados encontrados. No presente estudo, as palavras curtas freqüentemente receberam somente uma única fixação, com duração média de 540 milésimos de segundos. A primeira fixação em palavras curtas possibilitaria o acesso ao léxico ortográfico e a verbalização ocorreria de forma simultânea. Já nas palavras longas o tempo da primeira fixação diminuiu para 390 milésimos de segundos. Como na primeira fixação o acesso ao léxico ainda não seria suficiente para obter toda a informação necessária, o tempo diminui e o leitor apresenta outras fixações a fim de garantir a continuidade da decodificação da palavra (Sereno \& Rayner, 2003).

Os resultados de tempo total de fixação, encontrados em universitários brasileiros, diferiram consideravelmente dos estudos em língua inglesa (Starr \& Rayner, 200I). O tempo de fixação em língua inglesa dura aproximadamente 275 milésimos de segundos, enquanto os dados deste estudo variaram entre 300-800 ms. Essa diferença pode ser explicada em função de duas hipóteses: características distintas das línguas ou procedimentos diferentes nos estudos. Assim, a primeira hipótese poderia ser fortalecida pelo tamanho médio das palavras diferentes nas línguas ou do grau de transparência das línguas, sendo que a língua inglesa é composta de muito mais palavras irregulares do que a portuguesa. A alta incidência de ocorrência de palavras irregulares na língua inglesa tenderia a ser lida pela rota lexical (Ellis, 1993) enquanto que as palavras em português poderiam ser adequadamente lidas pela rota fonológica que é mais lenta. Outra hipótese diz respeito à diferença entre os tipos de provas usadas. Enquanto que os estudos ingleses analisam o tempo de fixação durante a leitura de textos, no presente estudo foram apresentadas palavras isoladas. A leitura de um texto pode ser facilitada devido ao contexto em que cada palavra está inserida (Rayner, Reichle \& Pollatsek, 2005), sendo possível deduzi-la e comprovar essa dedução mais rapidamente do que em palavras aleatórias, tal como as usadas neste estudo.
O número de fixações aumentou, como esperado, de acordo com o comprimento da palavra. Assim, quanto mais letras uma palavra tem, mais fixações são necessárias para sua leitura. Como em cada fixação é possível extrair a informação de um conjunto limitado de letras, palavras maiores demandarão mais fixações. Assim, tais resultados podem ser explicados em funções das características do span perceptual durante a leitura que, segundo Rayner e colaboradores (2005), é de aproximadamente 3 a 4 letras em bons leitores de língua inglesa. Desta for$\mathrm{ma}$, como as palavras longas tinham de 10 a 14 letras, mais fixações foram necessárias. A redução do span perceptual foi encontrada em leitores de línguas cuja ortografia é compacta, tais como Hebraico e Chinês (Rayner e cols., 2005).

Por fim, não foram observadas alterações no número de fixações com relação à regularidade dos itens. Tal ausência de efeito pode ser explicada pelo fato dos sujeitos serem todos universitários e com bom nível de leitura. De fato, o efeito de regularidade tem sido encontrado, para medidas de tempo de reação locucional, principalmente em crianças em fase inicial de alfabetização e diminuindo à medida que ocorre progresso nas séries escolares (Macedo, 1999; Pinheiro, 1995). Já as variáveis TTF e TPF apresentaram diferença entre palavras regra e irregulares. Estas duas variáveis de parâmetros de movimentos oculares parecem ser mais sensíveis ao efeito de regularidade do que aquelas de tempo de reação. Assim, o este estudo apresentou medidas de movimentos oculares como sendo mais sensíveis para o efeito de regularidade.

Para concluir, as propriedades de movimento ocular, durante leitura em português do Brasil, sofrem efeito de freqüência e tamanho das palavras apresentadas para leitura o que é condizente com os achados em outras línguas. O procedimento usado no presente estudo foi de leitura de palavra isolada e isto pode ter contribuído com o maior tempo de fixação e tempo total encontrado em leitores brasileiros. Novos estudos que apresentem textos, em vez de palavras isoladas, podem encontrar resultados similares àqueles verificados em outros idiomas. 


\section{Referências}

Abrams, R. A., \& Jonides, J. (1988). Programming saccadic eye movements. Journal of Experimental Psychology: Human Perception and Performance, 14, 428-443.

Balota, D. A. (1990). The role of meaning in word recognition. Em D. A Balota, G. B. Flores D’Arcais \& K. Rayner (Orgs.), Comprehension Processes in Reading (pp. 9-32). Hillsdale, NJ. Lawrence Erlbaum Associates.

Balota, D. A., \& Chumbley, J. I. (1984). Are lexical decisions a good measure of lexical access? The role of word frequency in the neglected decision stage. Journal of Experimental Psychology: Human Perception and Performance, 10, 340-57.

Blanchard, H. E., Pollatsek, A., \& Rayner, K. (1989). The acquisition of parafoveal word information in reading. Perception \& Psychophys, 46, 85-94.

Boyce, S. J., \& Pollatsek, A. (1992). Identification in objects in scenes: the role of scene backround in object naming.Journal of Experimental Psychology: Learning, Memory and Cognition, 18, 53 I-543.

Buswell, G. T. (1922). Fundamental reading habits: A study of their development. Chicago, IL: University of Chicago Press

Ellis, A. W. (1993). Reading, writing and dyslexia: A cognitive analysis. Hove, UK: Lawewnce Erlbaum Associates.

Hyönä, J., \& Olson, R. K. (1995). Eye fixation patterns among dyslexic and normal readers: effects of word length and word frequency. Journal of Experimental Psychology, 2I, I430- 1440.

Inhoff, A. W. (1984). Two stages of word processing during eye fixations in the reading of prose. Journal of Verbal Learning and Verbal Behavior, 23, 612-624.

Jacobs, A. M. (1987). On localization and saccade programming. Vision Research, 27, 1953-1966.

Kello, C. T., \& Plaut, D. C. (2003). Strategic control over rate of processing in word reading: a computational investigation. Journal of Memory and. Language, 48, 207-232.

Land, M. F. (2004). Eye movements in daily life. Em L. M. Chalupa \& J. S. Werner (Orgs.), The visual neurosciences (pp. I357-1368). Cambridge: The MIT Press.

Lemle, F. (|99|). Guia teórico do alfabetizador. São Paulo: Ática.

Macedo, E. C. (1999) Competência de leitura: Tecnologia na avaliação de leitura em voz alta, por crianças e adultos, de itens psicolingüísticos isolados. Tese de Doutorado, Universidade de São Paulo, São Paulo.
Macedo, E. C., Lukasova, K., Capovilla, F. C, Capovilla, A. G. S., \& Schwartzman, J. S. (2005). Movimentos oculares durante a leitura: implicações para a compreensão de modelos de processamento lingüístico. Em L. E. R. Valle. (Org.), Neuropsicologia e aprendizagem: para viver melhor. (pp. I25137). Ribeirão Preto: TecMedd.

MacKeben, M., Trauzettel-Klosinski, S., Reinhard, J., Dürrwächater, U., Adler, M., \& Klosinski, G. (2004). Eye movement control during single-word reading in dyslexics. Journal of Vision, 4, 388-402.

McConkie, G. W., \& Rayner, K. (1975). The span of the effective stimulus during an eye fixation in reading. Perception and Psychophysics, 17, 578-586.

McConkie, G. W., Zola, D., Grimes, J., Kerr, P.W., Bryant, N. R., \& Wolff, P. M. (I99I). Children's eye movements during reading. Em J. F. Stein (Org.), Vision and visual dyslexia (pp. 25I262). London: MacMillan Press.

Millet, S., \& Sparrow, L. (2004). Phonological codes are assembled before word fixation: evidence from boundary paradigm in sentence reading. Brain and Language, 90, 299-310.

Monaghan, J., \& Ellis, A. W. (2002). Age of acquisition and the completeness of phonological representations, Reading and Writing, 15, 759-788.

Murray, W. S, \& Kennedy, A. (1988). Spatial coding in the processing of anaphor by good and poor readers: evidence from eye movement analyses. Quarterly Journal of Experimental Psychology, 40A, 693-718.

Nazir, T. A., \& Jacobs, A. M. (199I). The effects of target discriminability and retinal eccentricity on saccade latencies: An analysis in terms of variable-criterion theory. Psychological Research, 53, 28I-289.

O'Regan, J. K. ( 1980). The control of saccade size and fixation duration in reading: The limits of linguistic control. Perception \& Psychophysics, 28, II2-II7.

Pinheiro, A. M. V. (1995). Reading and spelling development in Brazilian Portuguese. Reading and Writing, 7(I), I I I-I 38.

Pollatsek, A., \& Rayner, K. ( 982 ). Eye movement control in reading: the role of word boundaries. Journal of Experimental Psychology: Human Perception and Performance, 8, 817-833.

Pulvermüller, F., Assadollahi, R., \& Thomas, E. (200I). Neuromagnetic evidence for early semantic access in word recognition. European Journal of Neuroscience, 13, 201-205. 
Rayner, K. (1998). Eye movements in reading and information processing: 20 years of research. Psychological Bulletin, 124, $372-422$.

Rayner, K., Sereno, S. C., \& Raney, G. E. (1996). Eye movement control in reading: a comparison of two types of models. Journal of Experimental Psychology: Human Perception and Performance, 22(5), II88- 1200.

Rayner, K., Slowiaczek, M. L., Clifton, C., \& Bertera, J. H. (1983). Latency of sequential eye movements: Implications for reading. Journal of Experimental Psychology: Human Perception and Performance, 9, 912-922.

Rayner, K., Reichle, E. D., \& Pollatsek, A. (2005). Eye movement control in reading and the $E-Z$ Reader model. Em G. Underwood (Ed.), Cognitive processes in eye guidance (pp. I 31-162). Nova York: Oxford University Press.

Russo, I., \& Behlau, M. ( 1993) Percepção da fala: análise acústica do Português Brasileiro. São Paulo: Lovise.
Salthouse, T. A., \& Ellis, C. L. (1980). Determinants of eye-fixation duration. American Journal of Psychology, 93, 207-234.

Salthouse, T. A., Ellis, C. L., Diener, D. C., \& Sonberg, B. L. (I98I). Stimulus processing during eye fixations. Journal of Experimental Psychology: Human Perception and Performance, 7 , 611-623.

Sereno, S. C., \& Rayner, K. (1992). Fast priming during eye fixations in reading. Journal of Experimental Psychology: Human Perception and Performance, 18, I73-184.

Sereno, S. C., \& Rayner, K. (2003). Measuring word recognition in reading: eye movements and event-related potentials. Trends in Cognitive Sciences, 7, 489-493.

Starr, M. S., \& Rayner, K. (200I). Eye movements during reading: some current controversies. Trends in Cognitive Sciences, 5, I56-163.

Recebido em: 26/10/2006

Revisado em: 30/10//2007

Aprovado em: 05// I//2007

Sobre os autores

Elizeu Coutinho de Macedo (ecmacedo@mackenzie.br) - Doutor em Psicologia Experimental pela Universidade de São Paulo e Docente da Faculdade de Psicologia e Programa de Mestrado em Distúrbios do Desenvolvimento, Universidade Presbiteriana Mackenzie.

Katerina Lukasova - Mestre em Distúrbios do Desenvolvimento, Universidade Presbiteriana Mackenzie e docente da Faculdade de Psicologia, Universidade Cruzeiro do Sul.

Juliana Emy Yokomizo - Aluna de graduação de Faculdade de Psicologia, Universidade Presbiteriana Mackenzie.

Lívia Carolina Ariente - Aluna de graduação de Faculdade de Psicologia, Universidade Presbiteriana Mackenzie.

Juliana Koakutu - Aluna de graduação de Faculdade de Psicologia, Universidade Presbiteriana Mackenzie.

José Salomão Schwartzman - Doutor em Neurologia pela Universidade Federal de São Paulo e Docente da Faculdade de Psicologia e Programa de Mestrado em Distúrbios do Desenvolvimento, Universidade Presbiteriana Mackenzie.

Correspondência: Elizeu Coutinho de Macedo (ecmacedo@mackenzie.br)

Rua da Consolação, 876; Prédio Amantino Vassão, sala 62 - CEP: 01302-907

Apoio: Mackpesquisa, CNPq 